\title{
A SHORT PROOF OF A CONTINUED FRACTION TEST FOR THE STABILITY OF POLYNOMIALS
}

\author{
R. M. HOVSTAD
}

(Communicated by Paul S. Muhly)

\begin{abstract}
A generalized form related to the usual test fraction is used in an induction proof giving a quick access to the stability test for complex polynomials.
\end{abstract}

\section{INTRODUCTION}

We have the following definition of the stability of polynomials.

Definition. A complex polynomial

$$
p_{n}(z)=z^{n}+a_{n-1} z^{n-1}+\cdots+a_{0}
$$

for $n \geq 1$ is stable by definition if $p_{n}(z)=0$ implies $\operatorname{Re} z<0$. For background and further references, see [1, p. 283] and the original papers by H. S. Wall [2] and E. Frank [3]. Consider the fraction

$$
t_{n}(z)=\frac{\operatorname{Re}\left(a_{n-1}\right) z^{n-1}+i \operatorname{Im}\left(a_{n-2}\right) z^{n-2}+\cdots}{z^{n}+i \operatorname{Im}\left(a_{n-1}\right) z^{n-1}+\operatorname{Re}\left(a_{n-2}\right) z^{n-2}+\cdots} .
$$

This is the test fraction for the polynomial (1.1). We notice that the sum of the numerator and the denominator equals $p_{n}(z)$. The test fraction (1.2) is closely related to the test fraction in [1]. The difference is unimportant. See also [1, p. xxiii]. The fraction (1.2) is understood in the following way. Both in numerator and denominator the real parts and imaginary parts appear every second time until the process stops naturally with the last constant. The stability test then tells us that a polynomial (1.1) is stable if and only if the test fraction (1.2) can be written in the form

$$
\stackrel{\mathrm{K}}{\mathrm{K}=1}_{i t_{k}+d_{k} z}
$$

with $t_{k}$ real and $d_{k}>0$ for $1 \leq k \leq n$. We point out that standard polynomial division is used when checking that (1.2) can be written in the form (1.3). We

Received by the editors October 20, 1986 and, in revised form, December 8, 1987.

1980 Mathematics Subject Classification (1985 Revision). Primary 30B70; Secondary 30C10, $30 \mathrm{C} 15$. 
notice finally that the test fraction for the polynomial $(z+x+i y) p_{n}(z)$ with $x>0$ and $y$ real can be written

$$
\frac{1+(z / x+i y / x) t_{n}(z)}{z / x+i y / x+t_{n}(z)} .
$$

This is easily seen by observing that the denominator in the test fraction for this polynomial equals $(z+i y)\left(z^{n}+i \operatorname{Im}\left(a_{n-1}\right) z^{n-1}+\cdots\right)+x\left(\operatorname{Re}\left(a_{n-1}\right) z^{n-1}+\cdots\right)$ and adding that numerator plus denominator equals the polynomial. We will first prove by induction a lemma concerning a generalized form related to (1.4). This will next be used to prove the stability test by induction.

\section{The STABILITY TEST}

We will prove the following general lemma.

Lemma. Let $\alpha>0, \beta>0, u$ real, $v$ real, $r>0$ be given such that $(\alpha \beta / 4)$ $\times(u / \alpha-v / \beta)^{2}<r$. Then for all $t_{k}$ real and $d_{k}>0$ with $1 \leq k \leq n$ we have

$$
\frac{r+(\alpha z+i u) \mathbf{K}_{k=1}^{n}\left(1 /\left(i t_{k}+d_{k} z\right)\right)}{\beta z+i v+\mathbf{K}_{k=1}^{n}\left(1 /\left(i t_{k}+d_{k} z\right)\right)}={\underset{k=1}{n+1}}_{i T_{k}+D_{k} z},
$$

where $T_{k}$ is real and $D_{k}>0$ for $1 \leq k \leq n+1$.

Proof. The proof is by induction. But first, consider

$$
\frac{r+(\alpha z+i u) /\left(i t_{1}+d_{1} z+f\right)}{\beta z+i v+1 /\left(i t_{1}+d_{1} z+f\right)}=\frac{1}{i T_{1}+D_{1} z+F}
$$

We consider here all constants given and solve for $F$ with respect to $f$. We find

$$
F=\frac{q_{2} z^{2}+i q_{1} z+q_{0}+f\left(\left(\beta / r-D_{1}\right) z+i\left(v / r-T_{1}\right)\right)}{z\left(d_{1}+\alpha / r\right)+i\left(t_{1}+u / r\right)+f},
$$

where $q_{2}=(\beta / r) d_{1}-D_{1}\left(d_{1}+\alpha / r\right), q_{1}=(\beta / r) t_{1}+(v / r) d_{1}-T_{1}\left(d_{1}+\alpha / r\right)-$ $D_{1}\left(t_{1}+u / r\right), q_{0}=1 / r-(v / r) t_{1}+T_{1}\left(t_{1}+u / r\right)$. Now we introduce the numbers $\alpha_{1}, \beta_{1}, u_{1}, v_{1}$ by $\beta_{1}=d_{1}+\alpha / r, \alpha_{1} \beta_{1}=\alpha \beta / r^{2}, v_{1}=t_{1}+u / r, u_{1} / \alpha_{1}+v_{1} / \beta_{1}=$ $u / \alpha+v / \beta$. Choosing $D_{1}=\beta d_{1} / r \beta_{1}$ and $T_{1}=v / r-u_{1}$ we see that $q_{1}=q_{2}=0$. Denoting further $r_{1}=q_{0}$ in this case we observe that

$$
\frac{\alpha_{1} \beta_{1}}{4}\left(\frac{u_{1}}{\alpha_{1}}-\frac{v_{1}}{\beta_{1}}\right)^{2}=\frac{\alpha \beta}{4}\left(\frac{u}{\alpha}-\frac{v}{\beta}\right)^{2} \frac{1}{r^{2}}+r_{1}-\frac{1}{r}<r_{1} \text {. }
$$

We conclude finally that

$$
F=\frac{r_{1}+\left(\alpha_{1} z+i u_{1}\right) f}{\beta_{1} z+i v_{1}+f}
$$

with $\alpha_{1}>0, \beta_{1}>0, u_{1}$ real, $v_{1}$ real, $r_{1}>0$ such that

$$
\left(\alpha_{1} \beta_{1} / 4\right)\left(u_{1} / \alpha_{1}-v_{1} / \beta_{1}\right)^{2}<r_{1} \text {. }
$$


Thus the numbers $\alpha_{1}, \beta_{1}, u_{1}, v_{1}, r_{1}$ have the same properties as the numbers $\alpha, \beta, u, v, r$. Clearly also (2.2) implies (2.1). Next, the induction proceeds as follows. Let $n=1$. This case corresponds to $f=0$ in the above calculation. We can obviously use

$$
F=\frac{r_{1}}{\beta_{1} z+i v_{1}}=\frac{1}{i T_{2}+D_{2} z}
$$

from (2.2) with $T_{2}$ real and $D_{2}>0$. Putting this in (2.1) we conclude that the lemma is true for $n=1$ since also $T_{1}$ is real and $D_{1}>0$. Suppose that the lemma is true for $n=p \geq 1$. Consider therefore $n=p+1$. This case corresponds to

$$
f=\prod_{k=2}^{p+1} \frac{1}{i t_{k}+d_{k} z}
$$

in the above calculation. From the induction hypothesis and (2.2) we conclude that

$$
F=\prod_{k=2}^{p+2} \frac{1}{i T_{k}+D_{k} z}
$$

for suitable $T_{k}$ real and $D_{k}>0$ with $2 \leq k \leq p+2$. Using the result above we conclude from (2.1) that the lemma is true for $n=p+1$ since $T_{1}$ is real and $D_{1}>0$, and we are through. Next, we will use the lemma in the proof of the stability test.

Theorem (Stability test). A complex polynomial (1.1) of degree $n \geq 1$ is stable if and only if the test fraction (1.2) can be written in the form

$$
\stackrel{\mathrm{K}}{k=1}_{i t_{k}+d_{k} z}
$$

with $t_{k}$ real and $d_{k}>0$ for $1 \leq k \leq n$.

Proof. First, we take the simple part and suppose that the test fraction $t_{n}(z)$ in (1.2) can be written in the form (2.3). Clearly the numerator and the denominator in the test fraction cannot be both zero for any $z$ value. Particularly if $p_{n}(z)=0$ in $(1.1)$ for $\operatorname{Re} z \geq 0$ we see that $t_{n}(z)=-1$. On the other hand, taking fractions repeatedly in (2.3), we see that $\operatorname{Re} t_{n}(z) \geq 0$ or $t_{n}(z)$ is infinite. This is a contradiction and the polynomial in (1.1) is stable. Second, suppose that the polynomial in (1.1) is stable. We proceed by induction. Let $n=1$. In this case the test fraction can obviously be written in the required form (2.3). Suppose further that the test fraction for the polynomial in (1.1) with $n=q \geq 1$ can be written in the required form (2.3). Consider $n=q+1$. We shall therefore consider the polynomial $(z+x+i y) p_{q}(z)$ with $x>0$ and $y$ real. The test fraction for this polynomial has the form (1.4) for $n=q$. Using the induction hypothesis and appealing to the lemma with $r=1, \alpha=\beta=1 / x$, $u=v=y / x$ we conclude that the test fraction in question can be written in the required form (2.3) and we are through. 


\section{REFERENCES}

1. W. B. Jones and W. J. Thron, Continued fractions, analytic theory and applications, Encyclopedia of Mathematics and Its Applications, Vol. 11, Addison-Wesley, Reading, Massachusetts, 1980. Distributed now by Cambridge Univ. Press.

2. H. S. Wall, Polynomials whose zeros have negative real parts, Amer. Math. Monthly 52 (1945), 308-322.

3. E. Frank, On the zeros of polynomials with complex coefficients, Bull. Amer. Math. Soc. 52 (1946), 144-157.

NakkVes Vei 5 L 1020, Oslo 6, Norway 